\title{
MicroRNA-205 is downregulated in hepatocellular carcinoma and inhibits cell growth and metastasis via directly targeting vascular endothelial growth factor $A$
}

\author{
XUYA ZHAO, SHI ZHOU, DAZHI WANG, WEI HE, JUNXIANG LI and SHUAI ZHANG \\ Department of Interventional Radiology, Guizhou Cancer Hospital, Cancer Hospital of Guizhou Medical University, \\ Guiyang, Guizhou 550003, P.R. China
}

Received November 6, 2015; Accepted May 11, 2017

DOI: $10.3892 / \mathrm{ol} .2018 .8933$

\begin{abstract}
MicroRNAs (miRs) are an emerging class of non-coding, endogenous and small RNA molecules that serve important functions in tumorigenesis and development. The present study investigated the expression, functions and molecular mechanism underlying miR-205 in hepatocellular carcinoma. miR-205 was downregulated in hepatocellular carcinoma tissues and cell lines. Ectopic miR-205 expression suppressed hepatocellular carcinoma cell proliferation, migration and invasion in vitro. In addition, vascular endothelial growth factor A (VEGFA) was identified as a functional downstream target of miR-205 in hepatocellular carcinoma. Furthermore, knockdown of VEGFA revealed the same functions with miR-205 overexpression in hepatocellular carcinoma cells. These results provided evidence that miR-205 served important functions in the inhibition of hepatocellular carcinoma cells growth and metastasis via directly targeting VEGFA, which indicated that miR-205 may have therapeutic value for hepatocellular carcinoma.
\end{abstract}

\section{Introduction}

Hepatocellular carcinoma (HCC), a major subtype of primary liver cancer, represents the sixth most prevalent malignancy and the third most common cause of cancer-associated mortalities globally $(1,2)$. According to statistics, in 2015, 35,660 new HCC cases and 24,550 mortalities due to HCC were predicted in the USA (3). HCC initiation and progression involves a variety of risk factors, including chronic infection with hepatitis $\mathrm{C}$ virus or hepatitis $\mathrm{B}$ virus (HBV), alcohol or drug abuse and a high-fat or high-sugar diet (4). Among these

Correspondence to: Professor Shi Zhou, Department of Interventional Radiology, Guizhou Cancer Hospital, Cancer Hospital of Guizhou Medical University, 1 Beijing West Road, Yunyan, Guiyang, Guizhou 550003, P.R. China

E-mail: zhaoxy_327@126.com

Key words: hepatocellular carcinoma, vascular endothelial growth factor A, microRNA-205, metastasis, growth risk factors, infection with $\mathrm{HBV}$ is the most prevalent cause of the disease worldwide, and is responsible for the increasing incidence of HCC $(1,4)$. Developments have been made in improving diagnosis and therapeutic treatments for $\mathrm{HCC}$, but due to difficulties in diagnosis, a high frequency of recurrence and resistance to common chemotherapy and radiotherapy agents, the prognosis for patients with $\mathrm{HCC}$ remains poor (5-7). The 5-year survival rate is $<5 \%$ for patients with HCC and intra-hepatic or extra-hepatic metastasis (8). Therefore, it is urgent to elucidate the molecular mechanisms underlying HCC carcinogenesis and progression, and to develop effective strategies for the diagnosis, treatment and prognosis of HCC.

Previously, numerous studies have suggested that the abnormal expression of microRNAs (miRNAs/miRs) is associated with the carcinogenesis, progression, metastasis and recurrence of HCC $(9,10)$. miRNAs are an emerging class of non-coding, endogenous, small RNA molecules, 19-25 nucleotides in length, that negatively regulate the protein production of target mRNAs at the post-transcriptional level by interacting with the 3'untranslated regions (3'UTR) of target mRNAs $(11,12)$. In this manner, miRNAs were involved in various biological processes, including differentiation, proliferation, angiogenesis, metabolism, apoptosis, cell cycle and metastasis $(13,14)$. Accumulated evidence has suggested that miRNAs may act as a novel group of oncogenes or tumor suppressors, and the deregulation of miRNA and target mRNA expression levels may contribute to carcinogenesis and cancer development in a substantial number of human malignancies, including HCC (15-17). Therefore, further investigation concerning the expression levels, functions and molecular mechanisms underlying the effects of miRNAs in HCC will provide insight into the carcinogenesis and progression of HCC.

The aim of the present study was to investigate the expression levels, functions and molecular mechanisms underlying the effect of miR-205 in HCC. Initially, the expression levels of miR-205 in HCC tissues and cell lines were evaluated. Secondly, the effects of miR-205 on the proliferation and metastasis of HCC cells were determined by MTT assay, migration and invasion assays. Finally, vascular endothelial growth factor A (VEGFA) was identified as a functional downstream target of miR-205 in HCC. These results indicated that miR-205 may 
function as a tumor suppressor in HCC by directly targeting VEGFA, suggesting a potential targeted therapy for patients with HCC.

\section{Materials and methods}

Tissue specimens, cell culture and cell transfection. The present study was approved by the Ethics Committee of Guizhou Cancer Hospital (Guiyang, China). Written informed consent was obtained from all patients with HCC prior to enrollment in the present study. None of the patients had received antitumor treatment prior to surgery. HCC tissues and matched adjacent non-cancerous liver tissues were obtained from 32 patients (19 male; 13 female; age range, 46-74 years; mean age, 62 years) who underwent surgery at Guizhou Cancer Hospital between August 2013 and January 2015. All tissue specimens were immediately frozen and stored in liquid nitrogen.

HepG2, HuH-7, SMMC-7721 and BEL-7402 human HCC cell lines and L02 and HEK293T normal hepatic cell lines were purchased from the American Type Culture Collection (Manassas, VA, USA). All cell lines were maintained in Dulbecco's modified Eagle's medium supplemented with $10 \%$ fetal bovine serum (FBS), $100 \mathrm{IU} / \mathrm{ml}$ penicillin and $0.1 \mathrm{mg} / \mathrm{ml}$ streptomycin (all Gibco; Thermo Fisher Scientific, Inc., Waltham, MA, USA) at $37^{\circ} \mathrm{C}$ with $5 \% \mathrm{CO}^{2}$.

An miR-205 mimic and a negative control (NC) were purchased from Genepharm, Inc. (Sunnyvale, CA, USA); the miR-205 mimic sequence was 5'-UCCUUCAUUCCACCG GAGUCUG-3' and the NC sequence was 5'-UUCUCCGAA CGUGUCACGUTT-3'. VEGFA small interfering (si)RNA and NC siRNA were also purchased from Genepharm, Inc. The sequence for VEGFA siRNA and NC siRNA were as follows: VEGFA siRNA sense, 5'-GGCAGAAUCAUCACG AAGUTT-3' and antisense, 5'-ACUUCGUGAUGAUUCUGC CTT-3'; NC siRNA sense, 5'-UUCUCCGAACGUGUCACG UTT-3' and antisense, 5'-ACGUGACACGUUCGGAGAATT $-3^{\prime}$. miRNA and siRNA transfection was performed using Lipofectamine 2000 (Invitrogen; Thermo Fisher Scientific, Inc.), according to the manufacturer's protocol.

$R N A$ isolation and reverse transcription-quantitative polymerase chain reaction ( $R T-q P C R)$. Total RNA from tissues $(1 \mathrm{~g})$ or cells $\left(2 \times 10^{6}\right)$ was extracted using TRIzol reagent (Invitrogen; Thermo Fisher Scientific, Inc.) according to the manufacturer's protocol. A TaqMan miRNA assay (Applied Biosystems; Thermo Fisher Scientific, Inc.) was used for quantification of miR-205 expression levels, according to the manufacturer's protocol. For quantification of VEGFA mRNA expression levels, reverse transcription was performed using the M-MLV Reverse Transcription system (Promega Corporation, Madison, WI, USA). The temperature protocol for reverse transcription was as follows: $95^{\circ} \mathrm{C}$ for $2 \mathrm{~min}$; 20 cycles of $94^{\circ} \mathrm{C}$ for $1 \mathrm{~min}, 55^{\circ} \mathrm{C}$ for $1 \mathrm{~min}$ and $72^{\circ} \mathrm{C}$ for $2 \mathrm{~min}$; then $72^{\circ} \mathrm{C}$ for $5 \mathrm{~min}$. qPCR was performed using the SYBR-Green Master Mix (Takara Biotechnology Co., Ltd., Dalian, China). The thermocycling conditions for qPCR were as follows: $95^{\circ} \mathrm{C}$ for $10 \mathrm{~min}$, followed by 40 cycles of $95^{\circ} \mathrm{C}$ for $15 \mathrm{sec}$ and $60^{\circ} \mathrm{C}$ for $1 \mathrm{~min}$. U6 and $\beta$-actin were used as internal controls for miR-205 and VEGFA expression levels, respectively. The primers were as follows: miR-205, 5'-GCT
CCTTCATTCCACCGG-3' (forward) and 5'-CAGTGCAGG GTCCGAGGT-3' (reverse); U6, 5'-GCTTCGGCAGCACAT ATACTAAAAT-3' (forward) and 5'-CGCTTCACGAATTTG CGTGTCAT-3' (reverse); VEGFA, 5'-AACTTTCTGCTGTCT TGGGT-3' (forward) and 5'-TCTCGATTGGATGGCAGTA-3' (reverse); and $\beta$-actin, $5^{\prime}$-GGGACCTGACTGACTACCTC-3' (forward) and 5'-TCATACTCCTGCTTGCTGAT-3' (reverse). Each sample was analyzed in triplicate. Relative expression levels were calculated using the $2^{-\Delta \Delta C q}$ method (18).

MTT assay. The cell proliferation rate was evaluated using the MTT assay $(5 \mathrm{mg} / \mathrm{ml}$; Sigma-Aldrich; Merck KGaA, Darmstadt, Germany). HepG2 and HuH-7 cells were seeded into 96-well plates at a density of 3,000 cells per well. Following overnight incubation, miRNA or siRNA transfection was performed as aforementioned. MTT assays were performed following transfection at room temperature for 24, 48, 72 and $96 \mathrm{~h}$. In brief, $20 \mu \mathrm{l}$ MTT solution was added to each well. Following incubation with MTT solution at $37^{\circ} \mathrm{C}$ for an additional $4 \mathrm{~h}, 200 \mu \mathrm{l}$ dimethyl sulfoxide (Sigma-Aldrich; Merck $\mathrm{KGaA}$ ) was added to dissolve the formazan at $37^{\circ} \mathrm{C}$ for $10 \mathrm{~min}$. Finally, the absorbance at $490 \mathrm{~nm}$ was detected using an ELISA reader (BioTek Instruments, Inc., Winooski, VT, USA). Each sample was analyzed in triplicate.

Cell migration and invasion assays. Cell migration and invasion were evaluated using Transwell chambers (Corning Incorporated, Corning, NY, USA) with $8 \mu \mathrm{m}$ pore-size polycarbonate membranes. For the invasion assay, Transwell chambers were pre-coated with Matrigel (BD Biosciences, Franklin Lakes, NJ, USA). For the migration and invasion assays, $3 \times 10^{4}$ transfected HepG2 and HuH-7 cells suspended in $300 \mu \mathrm{l}$ serum-free DMEM were added to the upper chamber. DMEM (500 $\mu$ l) supplemented with 20\% FBS was added to the low chamber as a chemoattractant. Following incubation at $37^{\circ} \mathrm{C}$ for $48 \mathrm{~h}$, cells that had not migrated or invaded to the bottom surface of the Transwell chambers were carefully removed with cotton wool. The migrated and invaded cells were fixed with $100 \%$ methanol at room temperature for $10 \mathrm{~min}$, stained with $0.5 \%$ crystal violet for $10 \mathrm{~min}$ and imaged with a light microscope (magnification, x200).

Western blotting. Total protein was isolated from HepG2 and HuH-7 cells $\left(2 \times 10^{6}\right)$ using RIPA lysis buffer (Bioteke Corporation, Beijing, China) supplemented with $0.1 \mathrm{mg} / \mathrm{ml}$ phenylmethylsulfonyl fluoride, $1 \mathrm{mM}$ sodium orthovanadate and $1 \mathrm{mg} / \mathrm{ml}$ aprotinin. Protein concentration was determined using a bicinchoninic acid assay kit (Bioteke Instuments, Inc.). Total protein $(20 \mu \mathrm{g})$ was fractionated by $10 \%$ SDS-PAGE (Beyotime Institute of Biotechnology, Haimen, China), followed by transference to polyvinylidene (PVDF) membranes (Millipore, Bedford, MA). Non-specific binding sites of PVDF membranes were blocked using 10\% non-fat milk in Tris-buffered saline with $0.1 \%$ Tween (TBST) solution at room temperature for $1 \mathrm{~h}$. Subsequently, the membranes were probed with mouse anti-human VEGFA monoclonal primary antibody (dilution, 1:1,000; cat. no., ab155944;) and mouse anti-human GADPH monoclonal primary antibody (dilution, 1:1,000; cat. no., ab9484; both Abcam, Cambridge, UK). Following incubation overnight at $4^{\circ} \mathrm{C}$, membranes were 
A

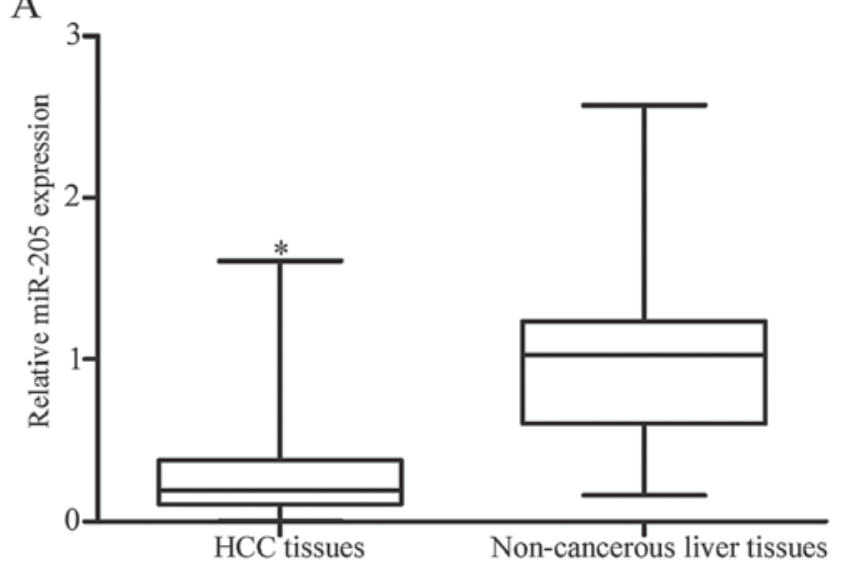

B

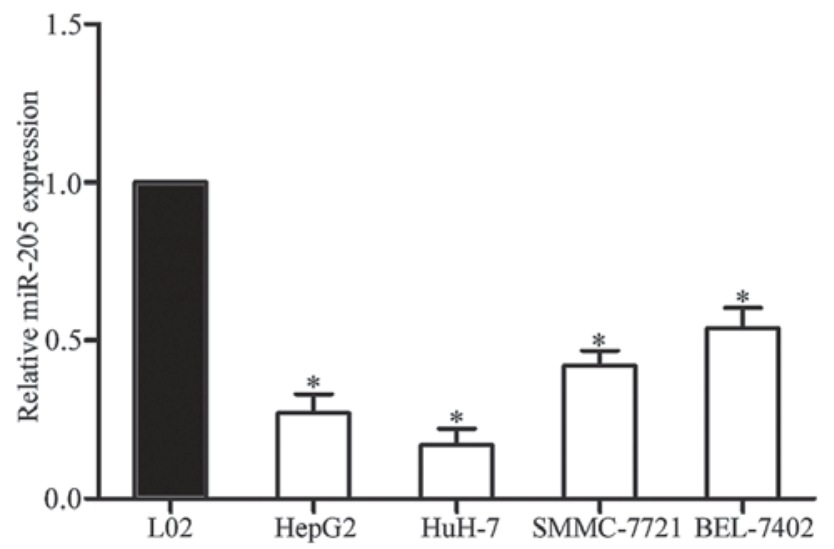

Figure 1. miR-205 expression levels were decreased in HCC tissues and cell lines. (A) Expression levels of miR-205 in HCC tissues and matched adjacent non-cancerous liver tissues were determined by reverse transcription-quantitative polymerase chain reaction. (B) Expression levels of miR-205 in HepG2, HuH-7, SMMC-7721 and BEL-7402 HCC cell lines and the L02 normal hepatic cell line. * $\mathrm{P}<0.05$ vs. respective controls. HCC, hepatocellular carcinoma; miR, microRNA.
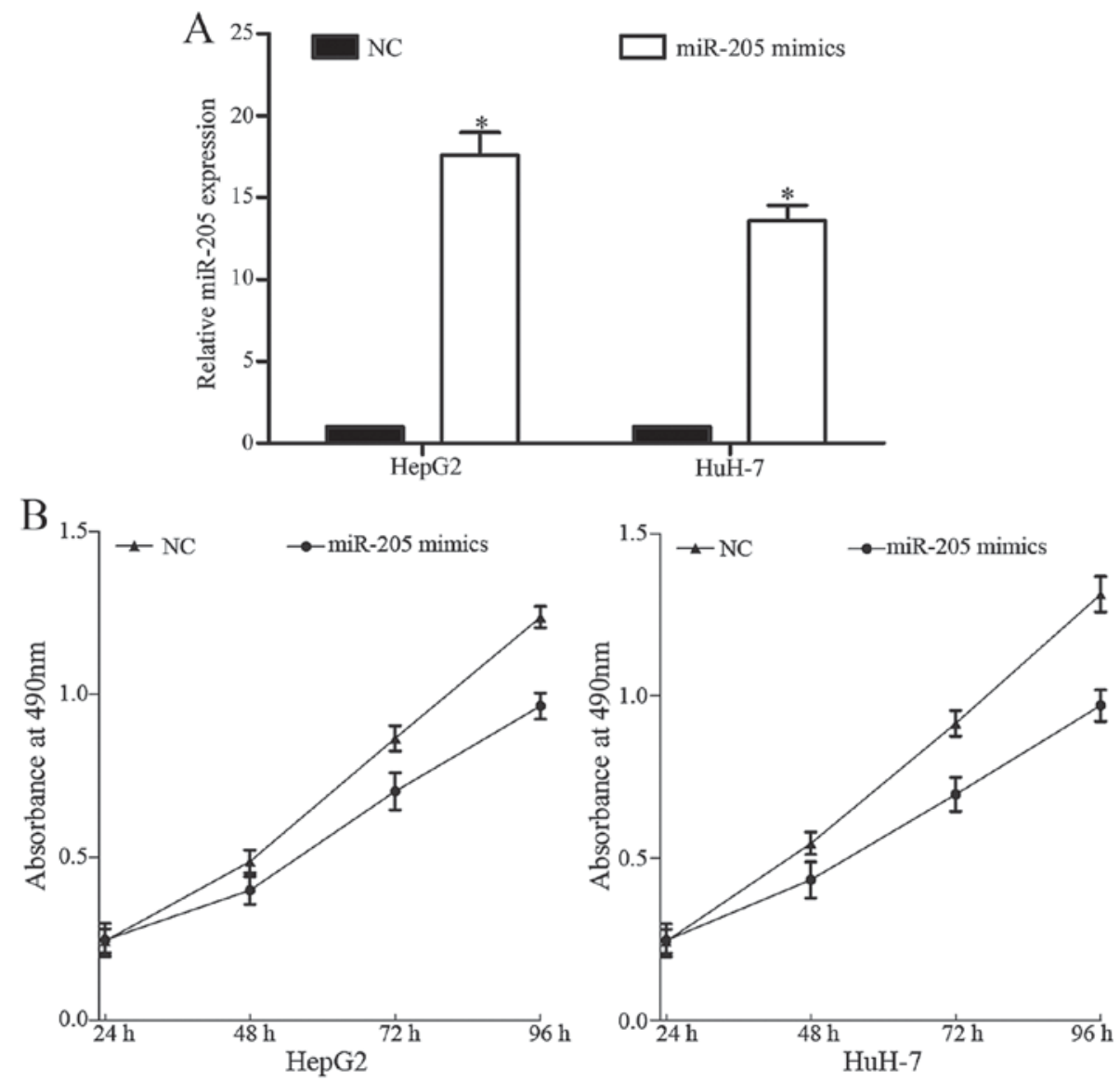

Figure 2. miR-205 inhibited the proliferation of HCC cells. (A) Expression levels of miR-205 in HepG2 and HuH-7 cells following transfection with miR-205 mimics or NC. (B) MTT assays were performed to determine the effect of miR-205 on the proliferation of HepG2 and HuH-7 cells. * $<0.05$ vs. NC. HCC, hepatocellular carcinoma. miR, microRNA; NC, negative control.

washed with TBST three times and incubated with goat anti mouse IgG horseradish peroxidase-conjugated secondary antibody (1:3,000 dilution; ab6789; Abcam) for $2 \mathrm{~h}$ at room temperature. Following washing three times with TBST, the membranes were visualized using enhanced chemiluminescence solution (Pierce; Thermo Fisher Scientific, Inc.).
Dual-luciferase reporter assay. The target genes of miR-205 were assessed using the miRNA target prediction tool TargetScan (version 6.0; http://www.targetscan. org/vert_60/) (19). In order to explore whether VEGFA was a direct target gene of miR-205, a dual-luciferase reporter assay was performed. HEK293T cells were co-transfected with 


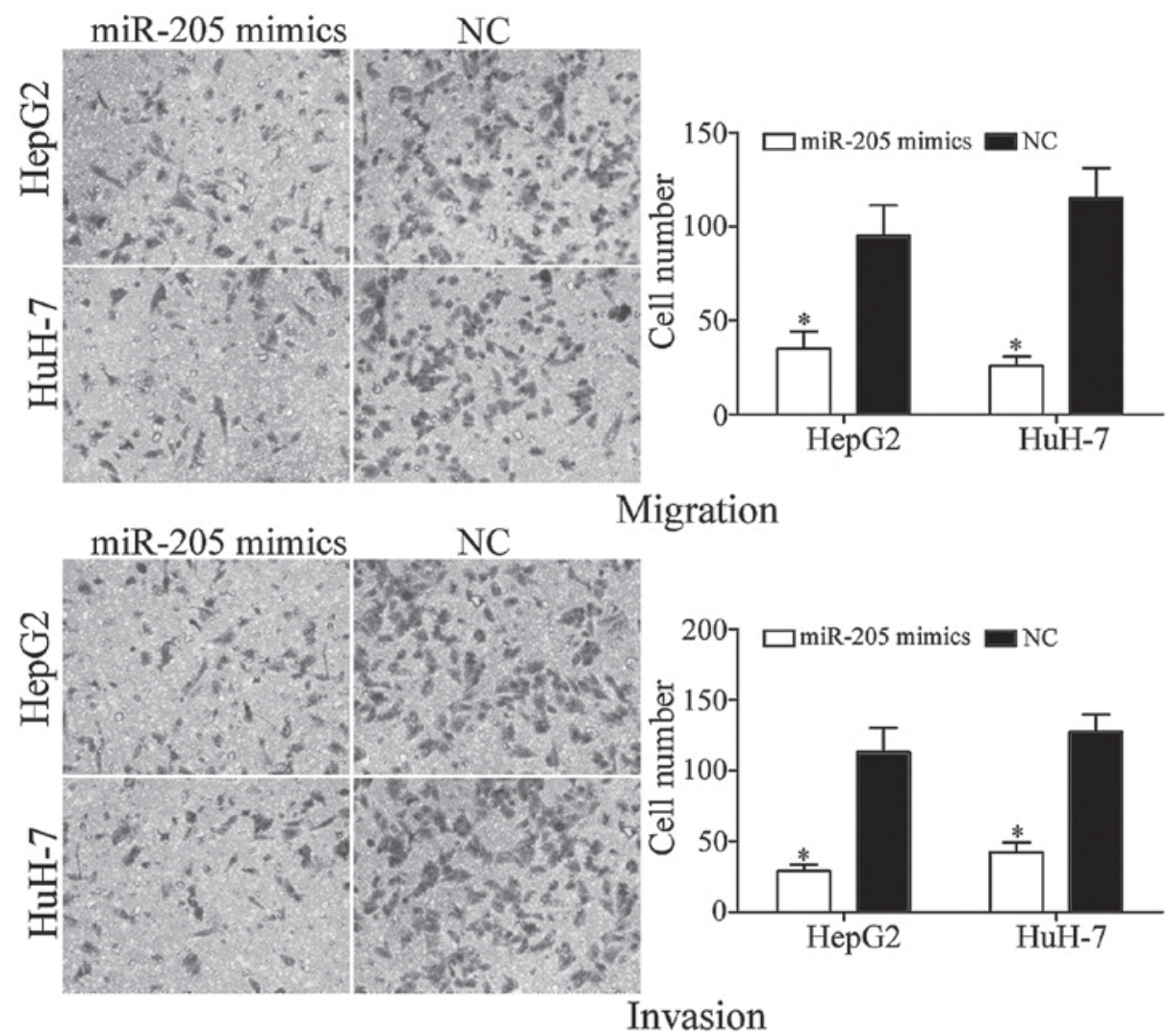

Figure 3. miR-205 overexpression suppressed the migration and invasion of HepG2 and HuH-7 cells (magnification, x200). "P<0.05 vs. NC. miR, microRNA; $\mathrm{NC}$, negative control.

VEGFA-3'UTR wild-type (Wt) or VEGFA-3'UTR mutant (Mut), miR-205 mimics or NC using Lipofectamine 2000 according to the manufacturer's protocol. Luciferase activities were evaluated $48 \mathrm{~h}$ following transfection using a Dual-Luciferase Reporter System (Promega Corporation, Madison, WI, USA). Renilla luciferase activity was detected as an internal control for firefly luciferase activity. Each sample was analyzed in triplicate.

Statistical analysis. Data were presented as the mean \pm standard deviation. Statistical analyses were performed using SPSS version 17.0 (SPSS Inc., Chicago, IL, USA). All statistical analyses were two-tailed. Data were analyzed using Student's t-tests and one-way analysis of variance, with Student-Newman-Keuls tests used to compare two groups in analyses with multiple groups. $\mathrm{P}<0.05$ was considered to indicate a statistically significant difference.

\section{Results}

miR-205 expression level was decreased in HCC tissues and cell lines. In order to determine miR-205 expression levels and its function in HCC, expression levels of miR-205 were detected in HCC tissues and matched adjacent non-cancerous liver tissues using RT-qPCR. As presented in Fig. 1A, miR-205 was significantly downregulated in HCC tissues compared with in matched adjacent non-cancerous liver tissues. In addition, miR-205 expression levels in HCC cell lines and a normal hepatic cell line were also determined. As presented in Fig. 1B, the expression levels of miR-205 were decreased in the four HCC cell lines compared with the L02 normal hepatic cell line. These results indicated that the downregulation of miR-205 may serve an important function in HCC carcinogenesis and progression.

miR-205 inhibited the proliferation of HCC cells. In order to investigate the functions of miR-205 in HCC, miR-205 mimics or $\mathrm{NC}$ were transfected into HepG2 and $\mathrm{HuH}-7$ cells. As presented in Fig. 2A, miR-205 was upregulated in HepG2 and HuH-7 cells following transfection with miR-205 mimics compared with NC, suggesting that HepG2 and HuH-7 cells were effective and adjustable models for the functional studies of miR-205.

Cell proliferation was evaluated using the MTT assay. miR-205 significantly reduced HepG2 and HuH-7 cell proliferation (Fig. 2B). These results indicated that overexpression of miR-205 inhibited the proliferation of HepG2 and HuH-7 cells.

MiR-205 inhibited migration and invasion of HCC cells. In order to investigate the function of miR-205 in metastasis, Transwell migration and invasion assays were performed. As presented in Fig. 3, upregulation of miR-205 induced the suppression of tumor cell migration and invasion in HepG2 and HuH-7 cells compared with NC controls. These results indicated that miR-205 suppressed HCC cell metastasis in vitro.

miR-205 directly targets the 3'UTR of VEGFA. In order to explore the molecular mechanism underlying the function of 
A

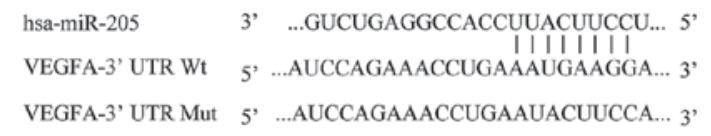

B

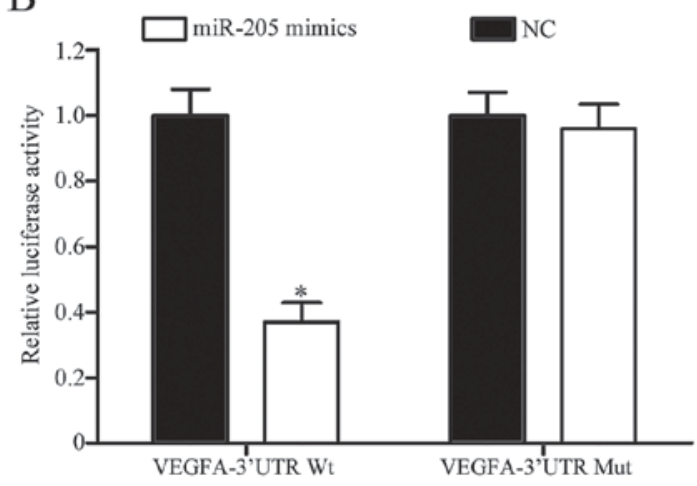

$\mathrm{C}$

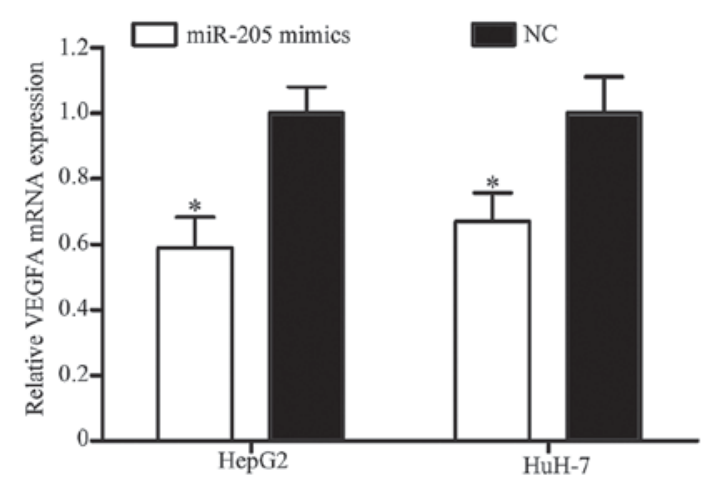

D

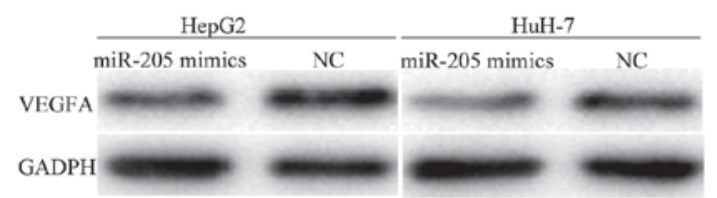

Figure 4. miR-205 directly targeted VEGFA by binding to its 3'UTR in hepatocellular carcinoma. (A) The predicted miR-205 binding site within VEGFA 3'UTR and its mutated version by site mutagenesis are presented. (B) Dual-luciferase reporter assays were performed to confirm the direct regulation of miR-205 in the 3'UTR of VEGFA. miR-205 decreased VEGFA expression at the (C) mRNA and (D) protein levels in HepG2 and HuH-7 cells. "P<0.05 vs. NC. miR, microRNA; VEGFA, vascular endothelial growth factor A; 3'UTR, 3'untranslated region; NC, negative control; Wt, wild-type; Mut, mutant.

miR-205 in HCC, TargetScan was used to predict the potential target genes of miR-205. As presented in Fig. 4A, VEGFA was identified to possess a putative miR-205 binding site in the 3'UTR. To verify whether VEGFA was a direct target gene of miR-205, a dual-luciferase reporter assay was performed. The results revealed that overexpression of miR-205 decreased the luciferase activity of VEGFA-3'UTR Wt, but did not decrease the luciferase activity of VEGFA-3'UTR Mut (Fig. 4B). Furthermore, RT-qPCR and western blotting was performed to investigate the effect of miR-205 on the expression levels of VEGFA mRNA and protein. As presented in Fig. 4C and D, upregulation of miR-205 significantly inhibited the expression level of VEGFA in HepG2 and $\mathrm{HuH}-7$ cells at the mRNA and protein levels, respectively. Taken together, these results indicated that VEGFA was a direct target of miR-205 in HCC.

VEGFA is involved in miR-205-induced suppression of $H C C$ cell proliferation, migration and invasion. To explore whether VEGFA acted as a critical mediator of miR-205 in HCC, VEGFA siRNA was used to knock down VEGFA expression. As presented in Fig. 5A, VEGFA was significantly downregulated in HepG2 and HuH-7 cells following transfection with VEGFA siRNA. Furthermore, MTT, migration and invasion assays were performed to evaluated the effect of VEGFA siRNA on the growth and metastasis of HCC. The results demonstrated that knockdown of VEGFA suppressed the proliferation, migration and invasion of HepG2 and HuH-7 cells (Fig. 5B and C). Together, these results suggested that miR-205 suppressed growth, migration and invasion of HCC by downregulating VEGFA.

\section{Discussion}

It is now widely accepted that miRNAs modulate various biological processes, including cancer initiation and progression. In the present study, miR-205 expression levels in HCC tissues and cell lines were determined, and the biological functions and molecular mechanisms underlying miR-205 in HCC carcinogenesis and progression were also investigated. It was revealed that miR-205 was downregulated in HCC tissues and cell lines. Overexpression of miR-205 significantly suppressed the proliferation, migration and invasion of HCC cells by directly targeting VEGFA. These findings suggested that miR-205 may be a notable tumor suppressor in HCC.

Previous studies have demonstrated that miR-205 functions as an oncogene in numerous types of human cancer (20-22). For example, in laryngeal squamous cell carcinoma, miR-205 was upregulated and enhanced cell growth and invasion via negative regulation of CDK2AP1 expression level (20). Expression levels of miR-205 were reported to be increased in endometrial cancer tissues (21). Kaplan-Meier survival analysis revealed that high expression levels of miR-205 were significantly associated with poor prognosis of patients with endometrial cancer (21). In addition, miR-205 promoted proliferation, metastasis and inhibited apoptosis of endometrial cancer cells by targeting the protein kinase B signaling pathway, phosphatase and tensin homolog (PTEN) and estrogen-related receptor $\gamma$ (23-25). In ovarian cancer, miR-205 was upregulated and miR-205 expression levels were associated with high pathological grade and advanced clinical stage of patients with epithelial ovarian cancer. Ectopic miR-205 expression improved migration and invasion abilities of ovarian cancer cells by directly targeting zing-finger E-box binding homeobox 1 (22). Lei et al (26) and Bai et al (27) investigated miR-205 expression levels in non-small cell lung cancer (NSCLC) tissues and cell lines. Upregulation of miR-205 increased the proliferation, migration, invasion and chemoresistance of NSCLC cells via regulation of the PTEN signaling pathway $(26,27)$. These results suggested 

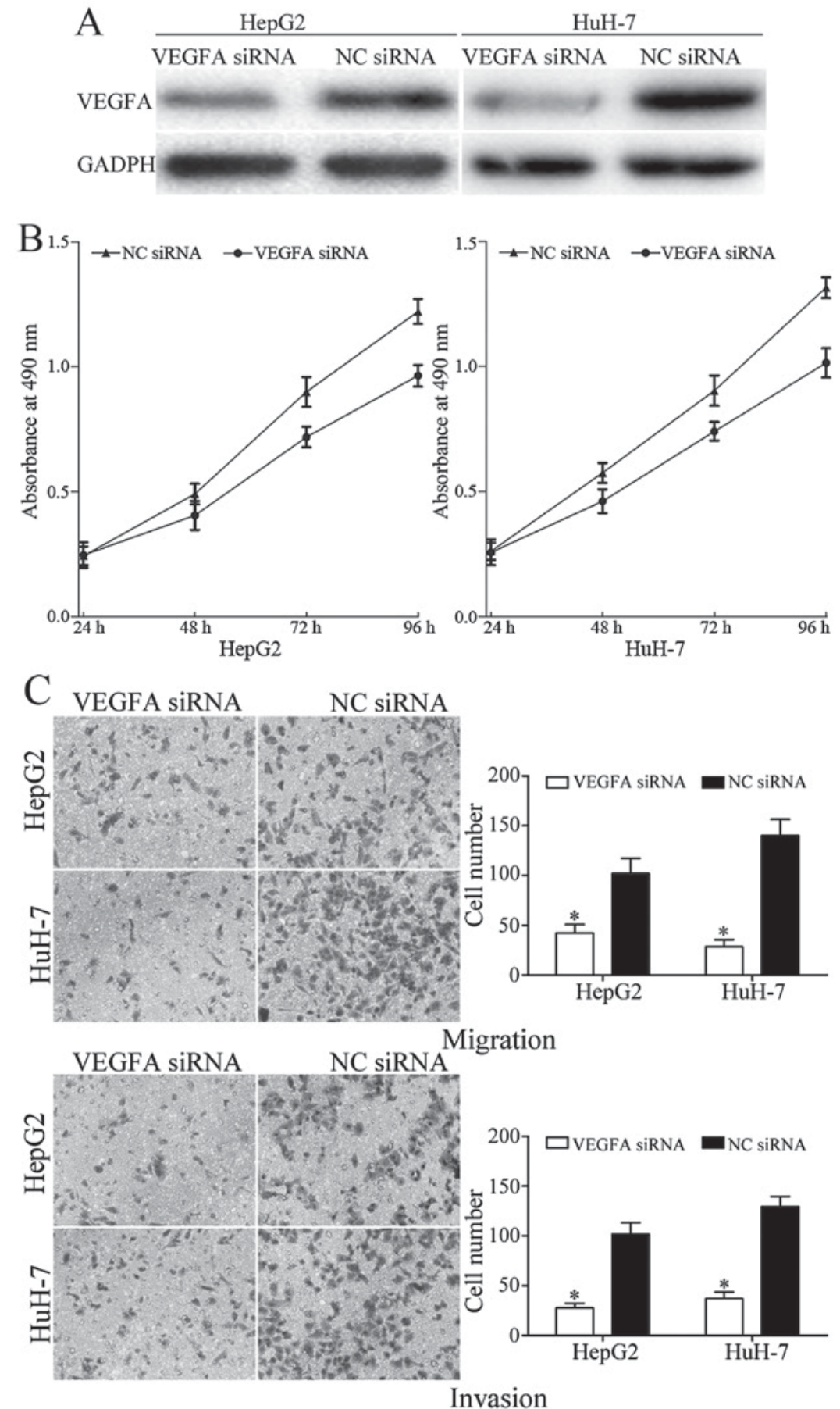

Figure 5. VEGFA was involved in miR-205-induced suppression of hepatocellular carcinoma proliferation, migration and invasion. (A) Protein expression levels of VEGFA in HepG2 and HuH-7 cells following transfection with VEGFA siRNA or NC siRNA. (B) MTT assays were performed to determine the effect of VEGFA siRNA on the proliferation of HepG2 and HuH-7 cells. (C) Knockdown of VEGFA suppressed migration and invasion of HepG2 and HuH-7 cells (magnification, x200). "P<0.05 vs. NC. VEGFA, vascular endothelial growth factor A; miR, microRNA; siRNA, short interfering RNA; NC, negative control.

that miR-205 may serve important functions in these types of cancer, and should be investigated as a potential therapeutic target for possible therapeutic strategies.

miR-205 has been reported to be a tumor suppressor in various types of cancer, including osteosarcoma (28), thyroid cancer (29), breast cancer (30,31), renal cell carcinoma (32), oral carcinoma (33) and prostate cancer (34). In accordance with these results, the present study indicated that miR-205 was downregulated in HCC tissues and overexpression of miR-205 inhibited HCC cell proliferation, migration and invasion in vitro. These conflicting results concerning miR-205 expression levels and functions demonstrate that miR-205 acted as an oncogene in certain types of cancer and as a tumor suppressor in others. This contradiction may be explained by the 'imperfect complementarity' of the interactions between miRNAs and target genes (35).

In order to understand the molecular mechanisms underlying miR-205-induced suppression of cell proliferation, migration and invasion in HCC, TargetScan and dual-luciferase reporter assays were performed. VEGFA was verified to be a 
direct target gene of miR-205 in HCC. Furthermore, RT-qPCR and western blotting were preformed to investigate whether miR-205 regulated VEGFA mRNA and protein expression levels. The results revealed that miR-205 significantly decreased VEGFA expression at the mRNA and protein levels. Taken together, miR-205 negatively regulated VEGFA expression by directly binding to the 3'UTR of VEGFA in HCC. Identification of the target gene of miR-205 is essential for understanding its functions in HCC carcinogenesis and progression. It is also important for developing novel therapeutic targets for HCC.

VEGFA, a 35-45 kD heparin-binding glycoprotein, is a key regulator of angiogenesis which is known to be a fundamental factor in the local growth of tumors and progression to metastasis (28). High expression levels of VEGFA have been reported in various types of human cancers, including HCC $(36,37)$. VEGFA serves an important function in tumor proliferation, migration, invasion and angiogenesis (38-40). Therefore, anti-VEGFA targeted therapy, including bevacizumab, has been widely used to treat cancers in a clinical setting (41). Regarding its cancer-associated functions in HCC, VEGFA is worth paying attention to as a potential target to treat patients with HCC. The present study revealed that miR-205 targeted VEGFA to suppress cell proliferation and metastasis in HCC. Collectively, miR-205/VEGFA based targeted therapy may be a novel therapeutic treatment for HCC.

In conclusion, the present study demonstrated that miR-205 was significantly downregulated in HCC tissues and cell lines, and overexpression of miR-205 inhibited the proliferation, migration and invasion of HCC cells by directly targeting VEGFA. These results help to further the understanding of the molecular mechanisms underlying HCC carcinogenesis and progression, and may potentially lead to novel targeted therapies for HCC in future studies.

\section{References}

1. El-Serag HB and Rudolph KL: Hepatocellular carcinoma: Epidemiology and molecularcarcinogenesis. Gastroenterology 132 2557-2576, 2007.

2. Ferlay J, Shin HR, Bray F, Forman D, Mathers C and Parkin DM: Estimates of worldwide burden of cancer in 2008: GLOBOCAN 2008. Int J Cancer 127: 2893-2917, 2010.

3. Siegel RL, Miller KD and Jemal A: Cancer statistics, 2015. CA Cancer J Clin 65: 5-29, 2015.

4. El-Serag HB: Hepatocellular carcinoma. N Engl J Med 365: $1118-1127,2011$

5. Wu Y, Cain-Hom C, Choy L, Hagenbeek TJ, de Leon GP, Chen Y, Finkle D, Venook R, Wu X, Ridgway J, et al: Therapeutic antibody targeting of individual Notch receptors. Nature 464: 1052-1057, 2010

6. Huang X, Qin J and Lu S: Up-regulation of miR-877 induced by paclitaxel inhibits hepatocellular carcinoma cell proliferation though targeting FOXM1. Int J Clin Exp Pathol 8: 1515-1524, 2015.

7. Yang LY, Fang F, Ou DP, Wu W, Zeng ZJ and Wu F: Solitary large hepatocellular carcinoma: A specific subtype of hepatocellular carcinoma with good outcome after hepatic resection. Ann Surg 249: 118-123, 2009.

8. Zhou YM, Zhang XF, Yu F, Liu XB, Wu LP, Li B and Yang JM: Efficacy of surgical resection for pulmonary metastases from hepatocellular carcinoma. Med Sci Monit 20: 1544-1549, 2014

9. Chen X, Bo L, Zhao X and Chen Q: MicroRNA-133a inhibits cell proliferation, colony formation ability, migration and invasion by targeting matrix metallopeptidase 9 in hepatocellular carcinoma Mol Med Rep 11: 3900-3907, 2015.

10. Zhang ZQ, Meng H, Wang N, Liang LN, Liu LN, Lu SM and Luan Y: Serum microRNA 143 and microRNA 215 as potential biomarkers for the diagnosis of chronic hepatitis and hepatocellular carcinoma. Diagn Pathol 9: 135, 2014.
11. Bartel DP: MicroRNAs: Genomics, biogenesis, mechanism, and function. Cell 116: 281-297, 2004.

12. Liu J: Control of protein synthesis and mRNA degradation by microRNAs. Curr Opin Cell Biol 20: 214-221, 2008.

13. Yates LA, Norbury CJ and Gilbert RJ: The long and short of microRNA. Cell 153: 516-519, 2013.

14. Hwang HW and Mendell JT: MicroRNAs in cell proliferation, cell death, and tumorigenesis. Br J Cancer 96 (Suppl): R40-R44, 2007.

15. Li B, Liu L, Li X and Wu L: miR-503 suppresses metastasis of hepatocellular carcinoma cell by targeting PRMT1. Biochem Biophys Res Commun 464: 982-987, 2015.

16. Li J, Fang L, Yu W and Wang Y: MicroRNA-125b suppresses the migration and invasion of hepatocellular carcinoma cells by targeting transcriptional coactivator with PDZ-binding motif. Oncol Lett 9: 1971-1975, 2015.

17. Zheng C, Li J, Wang Q, Liu W, Zhou J, Liu R, Zeng Q, Peng X, Huang C, Cao P and Cao K: MicroRNA-195 functions as a tumor suppressor by inhibiting CBX4 in hepatocellular carcinoma. Oncol Rep 33: 1115-1122, 2015.

18. Livak KJ and Schmittgen TD: Analysis of relative gene expression data using real-time quantitative PCR and the 2(-Delta Delta C(T)) method. Methods 25: 402-408, 2001.

19. Lewis BP, Burge CB and Bartel DP: Conserved seed pairing, often flanked by adenosines, indicates that thousands of human genes are microRNA targets. Cell 120: 15-20, 2005.

20. Zhong $\mathrm{G}$ and Xiong $\mathrm{X}$ : miR-205 promotes proliferation and invasion of laryngeal squamous cell carcinoma by suppressing CDK2AP1 expression. Biol Res 48: 60, 2015.

21. Karaayvaz M, Zhang C, Liang S, Shroyer KR and Ju J: Prognostic significance of miR-205 in endometrial cancer. PLoS One 7: e35158, 2012.

22. Niu K, Shen W, Zhang Y, Zhao Y and Lu Y: MiR-205 promotes motility of ovarian cancer cells via targeting ZEB1. Gene 574: 330-336, 2015.

23. Jin $\mathrm{C}$ and Liang R: miR-205 promotes epithelial-mesenchymal transition by targeting AKT signaling in endometrial cancer cells. J Obstet Gynaecol Res 41: 1653-1660, 2015.

24. Su N, Qiu H, Chen Y, Yang T, Yan Q and Wan X: miR-205 promotes tumor proliferation and invasion through targeting ESRRG in endometrial carcinoma. Oncol Rep 29: 2297-2302, 2013.

25. Zhang G, Hou X, Li Y and Zhao M: MiR-205 inhibits cell apoptosis by targeting phosphatase and tensin homolog deleted on chromosome ten in endometrial cancer Ishikawa cells. BMC Cancer 14: 440, 2014

26. Lei L, Huang Y and Gong W: miR-205 promotes the growth, metastasis and chemoresistance of NSCLC cells by targeting PTEN. Oncol Rep 30: 2897-2902, 2013.

27. Bai J, Zhu X, Ma J and Wang W: miR-205 regulates A549 cells proliferation by targeting PTEN. Int J Clin Exp Pathol 8: 1175-1183, 2015.

28. Wang L, Shan M, Liu Y, Yang F, Qi H, Zhou L, Qiu L and Li Y: miR-205 suppresses the proliferative and migratory capacity of human osteosarcoma Mg-63 cells by targeting VEGFA. Onco Targets Ther 8: 2635-2642, 2015.

29. Salajegheh A, Vosgha H, Md Rahman A, Amin M, Smith RA and Lam AK: Modulatory role of miR-205 in angiogenesis and progression of thyroid cancer. J Mol Endocrinol 55: 183-196, 2015.

30. Zhang $\mathrm{H}$ and Fan Q: MicroRNA-205 inhibits the proliferation and invasion of breast cancer by regulating AMOT expression. Oncol Rep 34: 2163-2170, 2015.

31. Zhang $\mathrm{H}, \mathrm{Li} \mathrm{B}$, Zhao $\mathrm{H}$ and Chang J: The expression and clinical significance of serum miR-205 for breast cancer and its role in detection of human cancers. Int J Clin Exp Med 8: 3034-3043, 2015.

32. Chen Z, Tang ZY, He Y, Liu LF, Li DJ and Chen X: miRNA-205 is a candidate tumor suppressor that targets ZEB2 in renal cell carcinoma. Oncol Res Treat 37: 658-664, 2014.

33. Kim JS, Park SY, Lee SA, Park MG, Yu SK, Lee MH, Park MR, Kim SG, Oh JS, Lee SY, et al: MicroRNA-205 suppresses the oral carcinoma oncogenic activity via down-regulation of Axin-2 in KB human oral cancer cell. Mol Cell Biochem 387: 71-79, 2014.

34. Wang N, Li Q, Feng NH, Cheng G, Guan ZL, Wang Y, Qin C, Yin CJ and Hua LX: miR-205 is frequently downregulated in prostate cancer and acts as a tumor suppressor by inhibiting tumor growth. Asian J Androl 15: 735-741, 2013.

35. Yu Z, Ni L, Chen D, Zhang Q, Su Z, Wang Y, Yu W, Wu X, Ye J, Yang S, et al: Identification of miR-7 as an oncogene in renal cell carcinoma. J Mol Histol 44: 669-677, 2013. 
36. Yamaguchi R, Yano H, Iemura A, Ogasawara S, Haramaki M and Kojiro M: Expression of vascular endothelial growth factor in human hepatocellular carcinoma. Hepatology 28: 68-77, 1998.

37. Miura H, Miyazaki T, Kuroda M, Oka T, Machinami R, Kodama T, Shibuya M, Makuuchi M, Yazaki Y and Ohnishi S: Increased expression of vascular endothelial growth factor in human hepatocellular carcinoma. J Hepatol 27: 854-861, 1997.

38. Zhuang Y and Wei M: Impact of vascular endothelial growth factor expression on overall survival in patients with osteosarcoma: A meta-analysis. Tumour Biol 35: 1745-1749, 2014.

39. Liu Y, Zheng Q, Wu H, Guo X, Li J and Hao S: Rapamycin increases pCREB, Bcl-2, and VEGF-A through ERK under normoxia. Acta Biochim Biophys Sin (Shanghai) 45: 259-267, 2013.
40. Wiszniak S, Mackenzie FE, Anderson P, Kabbara S, Ruhrberg C and Schwarz Q: Neural crest cell-derived VEGF promotes embryonic jaw extension. Proc Natl Acad Sci USA 112: 6086-6091, 2015

41. Hicklin DJ and Ellis LM: Role of the vascular endothelial growth factor pathway in tumor growth and angiogenesis. J Clin Oncol 23: 1011-1027, 2005. 